\title{
Reflexiones en torno a los entramados público-privados: el caso del polo farmacéutico del sur de la ciudad de Buenos Aires
}

María Soledad Arqueros Mejica

Doctora en Ciencias Sociales, Universidad de Buenos Aires; magíster en Economía Urbana, Universidad Torcuato Di Tella, y licenciada en Sociología, Universidad de Buenos Aires. Becaria posdoctoral del CONICET con sede en el Área de Estudios Urbanos del Instituto de Investigaciones Gino Germani, Universidad de Buenos Aires. Jefa de Trabajos Prácticos regular de la Carrera de Ciencias Ambientales de la Universidad Nacional de Avellaneda. soledad.arqueros@gmail.com.

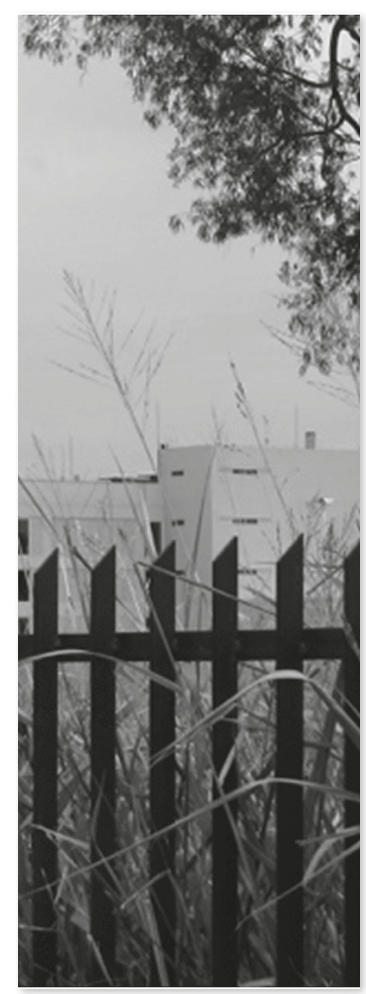

http://dx.doi.org/10.30972/crn.25253509 


\title{
Resumen
}

Este artículo indaga en las relaciones que establecieron las organizaciones estatales, empresariales y sociales en el marco del Polo Farmacéutico de Villa Soldati, las tensiones que surgieron en ese proceso y el modo en que afectaron dicha iniciativa. A pesar de haber contado con el apoyo del Ejecutivo local y de organismos del gobierno nacional y de ser promovido por un sector con poder económico e incidencia política, el proyecto del polo nunca llegó a concretarse. A partir del análisis, este trabajo evidencia que esas intervenciones se conforman en un entramado complejo de intereses, de disputas entre actores diversos y en contextos dinámicos, que inciden en las formas y orientación de los proyectos, así como en sus condiciones de posibilidad.

\section{Palabras clave}

Empresarialismo; Estado; territorio; desarrollo urbano; ciudad capitalista.

\begin{abstract}
Considerations regarding public-private sector frameworks: the case of the pharmaceutical pole to the south of the city of Buenos Aires.

This paper explores the relationships established between state, entrepreneurial and social organizations in Villa Soldati's Pharmaceutical Pole project. It considers the tensions that emerged during that process and the way in which they affected the initiative. Despite having the support of the local executive and some national government agencies, and being promoted by a sector with economic power and political influence, the Pharmaceutical Pole project never materialized. The analysis shows that these interventions are shaped in a complex web of interests, of disputes between diverse actors and in dynamic contexts, that affect the forms and orientation of these projects as well as their conditions of viability.
\end{abstract}

\section{Keywords}

Entrepreneurialism; State; Territory; Urban Development; Capitalist city. 


\section{Introducción}

Este artículo indaga en la trayectoria del Polo Farmacéutico de la Comuna 8, poniendo especial atención en las relaciones que establecieron las organizaciones estatales, empresariales y sociales, las tensiones que surgieron en ese proceso y el modo en que afectaron dicho proyecto. El polo se enmarcó en y constituyó uno de los hitos de la política de desarrollo de la Comuna 8 durante la década de 2000. Específicamente, fue presentado como una oportunidad para recuperar y dinamizar esta área "degradada” y deficitaria de la ciudad1. En palabras del entonces presidente de la Corporación Buenos Aires Sur², Enrique Rodríguez, "[La] instrumentación del Polo ha de cambiar todo el perfil de la zona” (Rodríguez, La Nación, 2005).

La propuesta surgió en el año 2004, en un contexto nacional de crecimiento y estabilidad económica, que repercutió a nivel local en un incremento en la renta productiva e inmobiliaria y una mayor recaudación fiscal (ArQ̨eros MEJICA, 2018). Estas señales fueron interpretadas por el Ejecutivo local como una oportunidad para lograr un mayor nivel de incidencia e interacción con el sector privado en la implementación de proyectos de "alto impacto territorial” (GCBA, 2004). La propuesta, que surgió de la cámara empresarial de laboratorios farmacéuticos nacionales COOPERALA, fue tomada por la Corporación Buenos Aires Sur. El polo se constituyó rápidamente en su principal proyecto, y recibió un fuerte apoyo del Ejecutivo local y de organismos nacionales, por lo que su concreción parecía inexorable.

Sin embargo, su trayectoria estuvo teñida por diversas dificultades, que incidieron en el curso, orientación y alcance del proyecto. En la actualidad, a catorce años de los primeros anuncios, solo uno de los once laboratorios involucrados construyó su planta. Los diez restantes, por el momento, desistieron de avanzar con las obras. Esto abre interrogantes sobre las tensiones que atraviesan este tipo de iniciativas y los condicionantes que inciden en su materialización. El presente artículo se sumerge en esa indagación y, desde ese lugar, busca aportar elementos para la comprensión de las formas de coordinación de la acción en el marco de los procesos de desarrollo urbano (Goicochea, 2014; Guevara, 2013; Cuenya y Corral, 2011, entre otros).

Para avanzar en esta investigación se realizaron entrevistas en profundidad a funcionarios estatales, empresas constructoras que participaron de las obras del polo, sindicalistas del sector farmacéutico y a referentes de las principales organizaciones involucradas en
1. La cuestión del desarrollo del sur (comunas 4 y 8 ) reingresó en la agenda estatal a mediados de la década de 1990, en el contexto de autonomización de la ciudad. A partir de entonces, se promovieron diversos proyectos y propuestas. El gran proyecto urbano Villa Olímpica, que se localiza en terrenos aledaños al polo farmacéutico, es una de las intervenciones más recientes impulsadas en el área (ARQueros MejICA, 2018).

\section{La Corporación Buenos} Aires Sur es una sociedad del Estado creada en el año 2000 con el propósito de impulsar el desarrollo de la zona sur de la ciudad. A lo largo de los años, esta entidad fue cambiando su papel, formas de gestión y atribuciones, a veces de manera formaly otras de modo informal, en un contexto que, parafraseando a PoRTEs (1999), podría caracterizarse como de amplia flexibilidad táctica. 
el conflicto. En paralelo, se analizaron los artículos periodísticos sobre el tema publicados entre los años 2004 y 2017 en medios específicos del sector (Pharmabiz y Pharma Baires), que son reconocidos desde ese ámbito como fuentes válidas de información y que tienen una notable circulación entre estos grupos. También se recurrió a notas publicadas por diarios nacionales, en algunas ocasiones escritas por funcionarios públicos con injerencia en el proyecto. Por último, el análisis se sostuvo en una diversidad de documentos, producidos por organizaciones estatales y no estatales en distintos momentos de este proceso de intervención.

El trabajo se divide en ocho apartados, cuya periodicidad fue definida en torno a los hitos del proceso que se analiza. El primero retoma algunos de los enfoques más influyentes en el estudio de las relaciones entre organizaciones estatales, empresariales y sociales en los procesos de crecimiento y transformación de las ciudades. El segundo se detiene en los actores que promovieron el polo farmacéutico, sus intereses y motivaciones en torno al proyecto. Los tres siguientes se focalizan en los diversos posicionamientos que asumieron esos actores en relación con el emprendimiento y cómo, en ese contexto, se fue definiendo un escenario de conflicto que tuvo efectos en el curso del proyecto. Los tres últimos apartados se concentran en el modo en que los cambios en el contexto político de la ciudad y en la coyuntura económica de los laboratorios afectaron la concreción del polo farmacéutico. Por último, se ofrecen algunas reflexiones que vinculan lo trabajado con los lineamientos teóricos que orientan el artículo.

\section{Los entramados público-privados en la investigación urbana}

Las relaciones entre los actores empresariales y estatales han sido ampliamente indagadas en la investigación. Entre las discusiones más recientes, se encuentran las de elitistas y pluralistas, que buscaron dar cuenta de la incidencia que tenían las elites y grupos de interés en la dinámica y orientación de la acción pública (Menazzi, 2015). Estas perspectivas tuvieron particular influencia en los estudios urbanos estadounidenses de las décadas de $1970 \mathrm{y}$ 1980, que a partir de entonces se interesaron en cómo incidían el poder, las jerarquías sociales y la acción de las elites en los procesos de desarrollo y transformación de las ciudades.

La tesis de la ciudad como máquina de crecimiento constituyó uno de los primeros esfuerzos en esta dirección. A partir del análisis de las formas de organización y coordinación 
de grupos, esta perspectiva puso de relieve el vínculo entre los negocios de base urbana, el desarrollo de las ciudades y las políticas públicas. Estos procesos se caracterizan por la articulación e interacción entre grupos del sector privado y del sector público, con el fin de asegurar las condiciones para el proceso de acumulación. Aunque se reconoce la emergencia de contracoaliciones, esta tesis sostiene que las "coaliciones para el crecimiento" tienen la capacidad de desarticularlas e imponerse (Logan y MoLотсн, 2015 [1984]). De esta manera, la lógica de la máquina de crecimiento parece inexorable, y estrecha la posibilidad de incorporar al análisis aspectos que escapen a las prioridades y requerimientos de los intereses que dominan la política local ${ }^{3}$. Esto supone la pérdida de autonomía del Estado local, que queda preso de los intereses de las elites (Cochrane, 1999).

La tesis de los regímenes urbanos ofrece una lectura más flexible, en tanto postula que las coaliciones para el crecimiento son solo una de las formas que puede asumir la coalición gobernante (CUENYA, 2005). El régimen urbano es definido como un conjunto de arreglos formales e informales que sirven de base para gobernar la ciudad. Este se conforma a partir de un entramado de intereses en el que las instituciones y los agentes públicos y privados negocian una agenda que guía las decisiones de gobierno (SтоNE, 1993 у 1980). Sin embargo, los regímenes urbanos pueden aparecer como completamente autónomos, puesto que se toma el contexto como dado en vez de como parte integrante del proceso social y político que se está analizando, y se independizan los actores y procesos locales de los sistemas de poder más amplios (Cochrane, 1999). Además, esta teoría se concentra en la acción de las elites y los sectores de poder, pero presta menos atención a los sectores que quedan fuera de esos grupos (Cochrane, 1999), que - como se pone de relieve en este artículo- tienen la capacidad de incidir en la política local.

En los últimos años ganó terreno la noción de empresarialismo urbano, que busca dar cuenta de la dinámica capitalista basada en un régimen de acumulación flexible. Desde esta perspectiva, la gobernanza urbana es entendida como una coalición amplia de fuerzas movilizadas por diversos agentes sociales, que excede al gobierno y la administración pública, que solo cumplen una función facilitadora y de coordinación. El empresarialismo urbano se distingue por la construcción de alianzas público-privadas para atraer fuentes de financiación e inversiones externas y nuevas fuentes de empleo; por el tipo empresarial de esas alianzas, que son de ejecución y diseño especulativos, y por estar centrado en la economía política del lugar más que en el territorio (HARvey, 2007). Desde esta perspectiva, la autonomía
3. Posteriormente, los autores de esta tesis flexibilizaron algunos de sus planteos iniciales y conservaron como elementos centrales los procesos de acción y las relaciones de poder entre actores (ver, por ejemplo, Моцотсн, 1999). Sin embargo, la difusión de los primeros escritos sobre las máquinas de crecimiento y la ausencia de traducciones al español de los nuevos trabajos contribuyeron a cierta persistencia en América Latina de las formulaciones originales de las máquinas de crecimiento. 
4. Podemos mencionar, entre otras, las investigaciones de GoIcochea (2014), GUEVARA (2013), y CUENYA (2006) sobre las diversas lógicas de los actores en torno al proyectos de refuncionalización y renovación urbana; los trabajos de RASPALL (2014), PANAIA (2004) y YUJNOVSKY y CLICHEvsKY (1979) sobre las características y reconfiguraciones de la

industria de la construcción; los estudios de MENAZZ (2015), Socoloff (2015),

CLICHEVSKY (1996), YUJNOSVKY

(1984), SCHTEINGART (1979) sobre las articulaciones entre el Estado y los empresarios inmobiliarios o de la construcción. Cabe señalar que varias de estas investigaciones tendieron a plantear enfoques originales para pensar las relaciones entre actores estatales y empresariales en los procesos de producción

de ciudad, en cuyo marco aportaron lecturas novedosas acerca de las realidades latinoamericanas. relativa del Estado local es aparente, puesto que la competencia interurbana fuerza a los distintos agentes a establecer configuraciones que en sí mismas constituyen la dinámica capitalista (HARVEY, 2007).

Las tres tesis reseñadas tuvieron una incidencia notable en los estudios urbanos latinoamericanos, que aportaron a la comprensión de los cambios en los modos de producción, circulación, apropiación y consumo del espacio, los procesos y dinámicas urbanas específicos que aquellos generaron y el papel de las organizaciones empresariales y estatales en esos procesos $^{4}$. Sin embargo, como sugieren otros trabajos, ni el Estado ni los empresarios son sectores monolíticos u homogéneos, las relaciones que establecen tampoco son necesariamente armónicas, y no siempre logran concretar los proyectos que impulsan.

Por un lado, la acción estatal no siempre es clara, coherente, ni racional (LASCOUMES y LE Galés, (2014); Oszlak y O’Donnell, 2008 [1976]; Thwaites Rey, 2005). Este artículo parte de la idea de que, por su esencia constitutiva, el Estado tiene como principal función garantizar la reproducción de la relación social capitalista y sus clases fundamentales, postulado que lleva a asumir su naturaleza compleja y contradictoria (ThwAITEs Rey, 2005). A su vez, la materialidad estatal - las instituciones y las burocracias públicas - no se produce de manera planificada (OszLAK, 2006), sino que es el resultado de los conflictos estructurales que se desarrollan en la sociedad, por lo que expresa las cristalizaciones de intereses diversos (BRown Y ERIE, 1984). Esto contribuye a que las agencias estatales tengan, en muchas ocasiones, diferentes posiciones sobre qué problemas son prioritarios, cuáles son las mejores maneras de encararlos y cómo avanzar en los procesos de intervención urbana.

Por otro lado, los empresarios tampoco constituyen un conjunto monolítico que maneja a su antojo el aparato estatal. Estos sectores pueden desplegar acciones divergentes o contradictorias en función de sus opciones particulares y, al mismo tiempo, están condicionados por otros intereses que inciden en su capacidad de organización y acción (BELTRÁn, 2011; Dossi Y Lissin, 2011; AcUÑA, 1994). A partir de los aportes de HARVEY (2007), puede decirse también que los empresarios pueden luchar por hacer su propia geografía histórica, pero lo hacen en circunstancias históricas y geográficas que no son de su elección individual, y que pueden favorecer o entorpecer la materialización de los proyectos que promueven. En particular, el territorio aparece como un ámbito en donde actores con intereses y motivaciones diversas disputan y negocian la producción, uso y apropiación de la ciudad, protagonizando una 
serie de conflictos que inciden en el curso y orientación de este tipo de proyectos (ARQuEROS Mejica, 2018; Svampa y Viale, 2014; RodríGueZ y Di Virgilio, 2011).

\section{Confluencias: los promotores del proyecto del polo farmacéutico}

El proyecto del polo farmacéutico se gestó en el año 2000 por impulso de la cámara de empresarios farmacéuticos nacionales COOPERALA. En el año 2004 se acordó con la Corporación Buenos Aires Sur (CBAS) emplazarlo en la Comuna 8 de la ciudad de Buenos Aires. Específicamente, se eligió localizarlo en terrenos de villa Soldati linderos a villa Lugano, con el argumento de que tenía potencialidad para reconvertir el perfil de esta zona, que concentra distintas villas y conjuntos de vivienda social ${ }^{5}$ (ver imagen 1). La iniciativa recibió el apoyo de diversas reparticiones públicas, de nivel local y nacional, que tenían distintos intereses en torno al polo.

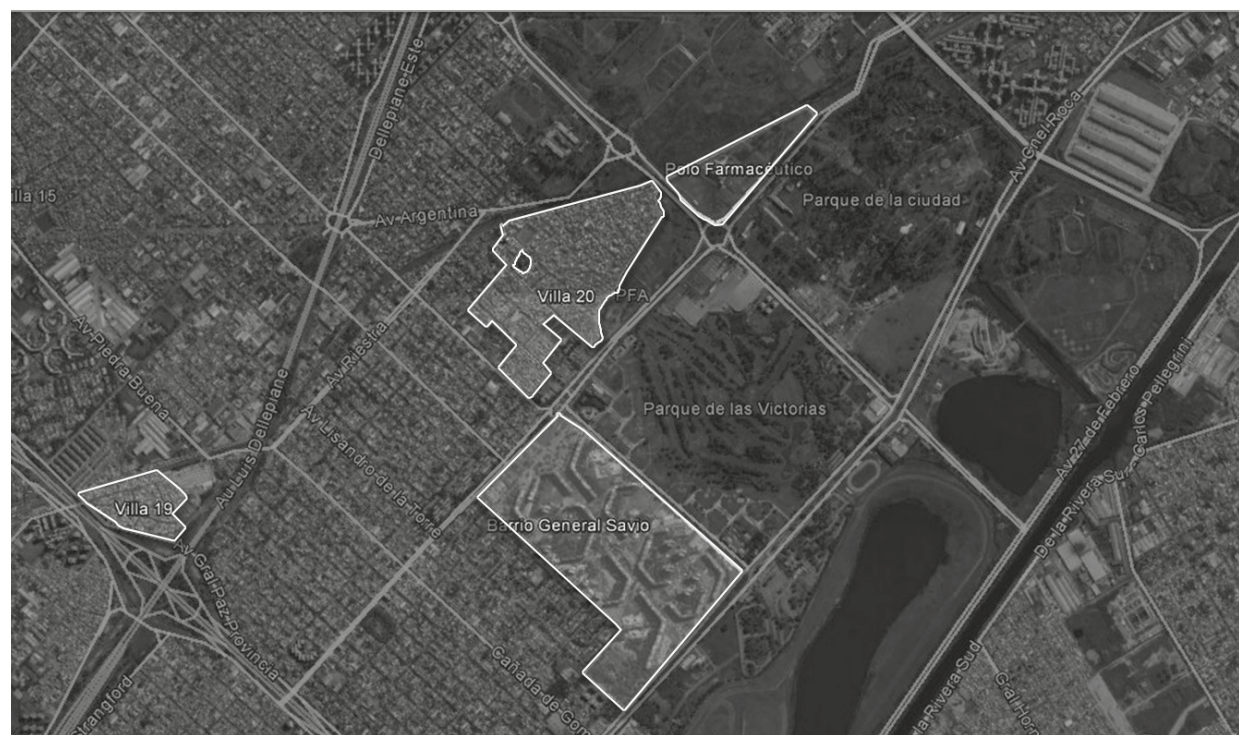

Imagen 1. Localización del polo farmacéutico en la Comuna 8. Fuente: elaboración propia en foto satelital del Google Earth del 9/9/2016

Cuaderno Urbano. Espacio, Cultura, Sociedad - Vol. 25 - N. ${ }^{\circ} 25$ (Noviembre de 2018)- Pp. 007-029 - ISNN1666-6186

\section{La Comuna 8}

históricamente aglutinó la mayor cantidad de población en villas de la ciudad y fue la localización tradicional de los conjuntos de vivienda social. Según datos del último Censo Nacional de Hogares, Población y Viviendas, la variación intercensal de la población en villas en esta área fue, entre los años 2001 y 2010, del 26,2 \%, superando por mucho el 13,7 $\%$ registrado a nivel de la comuna y el 3,9\% observado a escala de la ciudad. 
COOPERALA enmarcaba el proyecto en una estrategia para mejorar la competitividad de los laboratorios nacionales, que había sido afectada por la convertibilidad en la década de 1990. Si bien durante ese período el sector farmacéutico había crecido en producción y facturación, experimentó un aumento de la participación de los laboratorios extranjeros (AzPIAzu, 1999). Eso favoreció un proceso de fusiones y adquisiciones que dio lugar a una mayor concentración de la industria, que generó fuertes asimetrías de poder en el mercado interno (ABRUTZKY ET ÁL., 2015). Asimismo, en el año 2002, la Administración Nacional de Medicamentos, Alimentos y Tecnología Médica (ANMAT) adoptó un conjunto de regulaciones basadas en protocolos internacionales de fabricación y control de medicamentos. Para adecuarse a esas disposiciones los laboratorios debían ampliar y readecuar sus plantas de producción en un plazo de cinco años, cuestión que —según argumentaban— resultaba imposible en sus localizaciones originales.

La iniciativa fue recibida con interés por la Corporación Buenos Aires Sur (CBAS), que es una empresa del Estado creada en el año 2000 con el fin promover el desarrollo "humano, económico y urbano” de la zona sur de la ciudad (Ley 490 de 2000). A partir del año 2003, esta organización adoptó la estrategia de crear "Polos de Desarrollo” para avanzar con ese propósito. Las zonas de villa Soldati y villa Lugano resultaban particularmente atractivas para emplazar ese tipo de proyectos, puesto que concentraban grandes extensiones de terrenos de dominio privado de la ciudad. En este contexto, el proyecto del polo farmacéutico fue presentado como una oportunidad para impulsar la transformación del sudoeste de la ciudad, sumarle dinamismo y generar fuentes de empleo, a través de la promoción de un sector industrial con buena proyección en el mercado local e internacional.

El gobierno local estaba interesado en retener a la industria farmacéutica, en un contexto en el que buscaba recuperar el Producto Bruto Geográfico (que se había contraído como consecuencia de la crisis de 2001) y reposicionar a la ciudad en el mercado internacional. El sector farmacéutico tenía una presencia importante en la ciudad y un peso creciente en la industria manufacturera local; era una de las que presentaba la mayor cantidad de personal asalariado y de las que tenía mejores perspectivas de crecimiento. Además, a diferencia de otras industrias, tenía la posibilidad de incorporar tecnologías que permitieran su funcionamiento en un tejido urbano consolidado.

El proyecto también recibió el apoyo de las agencias de ciencia y técnica de nivel nacional, como la Agencia de Promoción Científica y Tecnológica de la Nación, primero, 
y el Ministerio de Ciencia y Técnica de la Nación, a partir del año 2008. Estos organismos estaban interesados en la promoción de la Investigación Científica, el Desarrollo y la Innovación Tecnológica ( $+\mathrm{D}$ ) del sector, que presentaba históricamente un déficit comercial debido a la importación de productos activos y su perfil manufacturero. Así, en 2006, se conformó una asociación ad hoc entre el Fondo Tecnológico Argentino (FONTAR), COOPERALA, la Facultad de Farmacia y Bioquímica de la Universidad de Buenos Aires, la Universidad Abierta Interamericana, la Fundación Pablo Cassará, la CBAS, el Ministerio de Salud de la Nación y la Administración Nacional de Laboratorios para desarrollar un conjunto de acciones, entre las que se incluía la puesta en funcionamiento del polo farmacéutico.

\section{Los primeros avances del proyecto del polo farmacéutico}

El proyecto tomó estado público en el año 2004, cuando el jefe de Gobierno Aníbal Ibarra (2000-2005) solicitó en la Legislatura porteña la rezonificación de los terrenos para habilitar su emplazamiento. En los días previos a la sesión, COOPERALA publicó solicitadas en diarios de tirada nacional que pedían la "resolución rápida de la rezonificación solicitada por el Jefe de Gobierno, Aníbal Ibarra, para un predio de villa Soldati en el que se prevé instalar un polo farmacéutico". Allí argumentaba que "los laboratorios tienen un plazo perentorio para montar sus plantas de fabricación de acuerdo con nuevas exigencias técnicas de la autoridad sanitaria, y prevén hacer esta adecuación cuando trasladen sus instalaciones a ese predio" (La Nación, 11 de octubre de 2004). Los miembros de COOPERALA estuvieron presentes en la sesión legislativa alentando a quienes se posicionaron a favor y abucheando a quienes estuvieron en contra.

Los legisladores tuvieron posturas divididas. Quienes apoyaron el proyecto manifestaron que revalorizaría el área sur y revertiría el proceso de desindustrialización iniciado durante la última dictadura militar (1976-1983). Valoraron el carácter público-privado del emprendimiento, la inversión que involucraba y su potencialidad para crear empleo. Los diputados que estuvieron en contra señalaron que el polo crearía una barrera urbana en la zona, que acentuaría la segregación y desintegración urbanas. Sostuvieron que el proyecto implicaba la privatización de terrenos que eran de dominio público y que promovía al mercado como planificador urbano. Denunciaron también que el polígono designado para 
6. Este procedimiento implica una primera aprobación en la Legislatura, la realización de una audiencia pública y posteriormente su tratamiento en segunda lectura en la Legislatura para su sanción definitiva.

7. Esta clasificación no está exenta de tensiones. Tal como se desprende de este artículo, algunos sectores "no estatales" tienen acceso al aparato estatal y capacidad de incidencia en el rumbo de los procesos políticos. A su vez, la trayectoria de varios de los agentes que ocupan cargos públicos hace que esta separación sea poco nítida, en particular teniendo en cuenta los procesos de circulación, que permiten que algunos empresarios ocupen cargos estratégicos en la administración pública $y$ viceversa (GAGGERO E IRAMAIN, 2016). En trabajos anteriores, dimos cuenta de que esa circulación es observable también entre organizaciones sociales de base territorialy agencias estatales (ARQUEROS MEIICA, 2014). el emprendimiento incluía terrenos de la policía federal, que pocos meses antes habían sido dispuestos por el instituto de la vivienda para la urbanización de la villa 20 (ver imagen 1). La ley resultó aprobada en primera lectura ${ }^{6}$.

\section{Intereses en conflicto}

El avance producido en el ámbito legislativo movilizó a actores estatales y no estatales ${ }^{7}$, que tenían intereses y posturas diversas con respecto al proyecto, y definió un escenario de conflicto. Esa disputa se expresó en el aparato del Estado, puesto que el Instituto de la Vivienda de la Ciudad (IVC) había diseñado un proyecto para la reurbanización de villa 20 que incluía esos terrenos. La sanción en primera lectura de la ley del polo produjo malestar entre el personal de ese organismo, que proponía construir ahí viviendas nuevas para relocalizar familias. De hecho, había realizado varias gestiones ante el gobierno nacional, que era el propietario de las tierras, para obtener su titularidad y destinarlas a dicho fin. La CBAS argumentó que para construir en esos terrenos era necesario realizar un estudio de suelo, que demoraría el proyecto de urbanización, y que por ese motivo era preferible destinarlos al polo. Ofreció, a cambio, venderle cuatro hectáreas de un predio lindero localizado en el Parque de las Victorias (ver imagen 1). Después de varias discusiones, el IVC aceptó la oferta.

Sin embargo, el conflicto se mantuvo a nivel territorial, ya que las organizaciones y habitantes de la Comuna 8 también tenían intereses y posicionamientos diversos. Los habitantes de villa 20 rechazaron el principio de acuerdo entre el IVC y la CBAS, y destacaron su lucha histórica para que esos terrenos fueran afectados a la urbanización del barrio. A su vez, el Foro Social de Salud de Capital zona oeste y el Movimiento por la Construcción del Hospital de Lugano señalaron que parte de esas tierras estaban destinadas a la construcción de un hospital, que los habitantes de la zona reclamaban desde la década de 1980. Estos grupos cuestionaron a los organismos públicos por haberlos mantenido al margen del proyecto y de las negociaciones con la CBAS, y reclamaron su participación en el proceso de toma de decisiones. Plantearon dudas con respecto a la generación de fuentes de trabajo y expresaron su preocupación por el impacto y contaminación que podría generar el polo.

El sector farmacéutico estaba alineado con el proyecto, pero prevalecieron diferentes intereses. Por un lado, los laboratorios remarcaban la necesidad de expandirse y readecuar sus plantas, así como la necesidad de mejorar la competitividad para exportar y competir 
con empresas extranjeras. Diferenciaron el proyecto de las políticas neoliberales, con el argumento de que la concreción del polo permitiría avanzar en la "soberanía farmacéutica". Sostuvieron que los laboratorios iban a pagar por los terrenos, invertir en el país y generar empleos, lo que contribuiría a la resolver la desigualdad. Por el otro lado, el gremio Asociación de Trabajadores de la Sanidad Argentina señaló que la falta de adecuación de los laboratorios a la normativa vigente estaba produciendo el cierre y migración de algunos laboratorios al conurbano o el exterior, y generaba desocupación entre los empleados del sector. Solicitaron a la CBAS la participación del sindicato en el proyecto para asegurar que se mantendrían los puestos de trabajo y evitar la precarización laboral.

\section{Profundización del conflicto y redefiniciones}

Esa compleja trama de intereses se fue profundizando en los meses siguientes. En este sentido, en el año 2005, continuaron produciéndose intercambios entre las organizaciones estatales y no estatales, se sumaron a la discusión grupos que no estaban involucrados originalmente y se produjeron reposicionamientos entre los que sí lo estaban. Esto dio lugar a un repertorio de acciones que incidió en el curso del proyecto.

Durante ese año el director de la CBAS, Enrique Rodríguez, escribió notas en los principales diarios del país defendiendo la articulación público-privada como estrategia de desarrollo y promocionando el proyecto del polo farmacéutico (La Nación, 12 de mayo de 2005). En paralelo, la corporación promovió negociaciones entre las organizaciones estatales y los grupos que se oponían al polo y presionó a la Legislatura para que tratara en segunda lectura el proyecto de ley que habilitaba dicha intervención.

En este contexto, la CBAS promovió un acuerdo con dos de las principales cooperativas de villa 20, en el cual proponía adjudicarles la construcción de viviendas enmarcadas en la urbanización del barrio, a cambio de que cedieran la presión sobre los terrenos ambicionados para el polo. Como resultado de ese proceso, la corporación, Cooperala, el Instituto de la Vivienda, representantes locales del Ejecutivo y el Legislativo y las dos organizaciones de villa 20 firmaron un acta de acuerdo. Una de las cooperativas firmantes se retractó a partir del repudio de los habitantes del barrio, mientras que la otra se mantuvo firme en su postura. Esto produjo una división entre los habitantes de la villa, ya que un grupo rechazaba y el otro aceptaba —e incluso promovía — el proyecto del polo. 
Esas divergencias se expresaron en la Legislatura, en el tratamiento en segunda lectura el proyecto de rezonificación de los terrenos para el emplazamiento del polo. Específicamente, cuando comenzó a debatirse el proyecto se desarrolló una fuerte discusión entre los empresarios farmacéuticos, los habitantes de la villa 20 que aceptaban el polo, aquellos que lo rechazaban y los legisladores que estaban a favor y en contra, lo que provocó la suspensión de la sesión y la intervención del personal policial.

En paralelo, habitantes de villa Lugano presentaron varios recursos de amparo que solicitaban el cese de la construcción de viviendas en el Parque de las Victorias. El juez a cargo ordenó una medida cautelar, y pocos días después realizó una inspección en compañía de habitantes de la villa 20, representantes del IVC, la CBAS, el asesor tutelar de la ciudad y personal de la Subsecretaría de Planeamiento del Gobierno de la Ciudad. En este contexto, se decidió ampliar la medida afectando a todas las licitaciones "que bajo concepto de urbanización, construcción de viviendas y/o instalación de polos industriales, tecnológicos u homólogos" pudieran comprometer las zonas abarcadas por el polo y la villa 20, entre otras (Poder Judicial de la Ciudad de Buenos Aires, 2005).

El GCBA y la corporación apelaron la medida. Argumentaron que el fallo era excesivo, que el proyecto todavía se estaba tratando en la Legislatura, que en una parte de los terrenos afectados se estaban construyendo viviendas para la población de villa La Dulce y que la cautelar causaba un gravamen al Estado, debido a que el contratista de las obras podía reclamarle dinero por los daños y perjuicios ocasionados. Pocos días después se presentó en el juzgado un habitante de villa La Dulce para explicar las diferencias que existían entre las obras de vivienda para los habitantes de su barrio y el conjunto de emprendimientos impulsados por el Ejecutivo local en el área, y solicitó que se consideraran los derechos de las familias afectadas por la suspensión de las obras. También se presentaron representantes de dos de las cooperativas de la villa 20 , que solicitaron que se levantara la medida cautelar debido a que impedía la realización de obras de saneamiento en dos manzanas del barrio.

Para destrabar este conflicto se ampliaron las negociaciones con los grupos involucrados. Esto implicó cierto desplazamiento del polo como centro de la cuestión, para integrar también la reurbanización de la villa 20 y la construcción del Hospital General de Agudos de Villa Lugano. Específicamente, se generó un acuerdo en la Legislatura para sancionar, durante la 
misma sesión de agosto de 2005, tres leyes, una por cada uno de estos proyectos: la Ley 1768 del Polo Farmacéutico; la Ley 1769 de construcción del Hospital de Villa Lugano y La ley 1770 de reurbanización de la villa 20.

\section{Cambio de contexto y demoras}

En los meses siguientes a la sanción de la Ley del Polo Farmacéutico, el contexto político de la ciudad se modificó significativamente. En marzo de 2006 el jefe de Gobierno Aníbal Ibarra, que había sido uno de los principales promotores del proyecto, fue destituido y reemplazado en su cargo por Jorge Telerman (2005-2007). El cambio de autoridades tuvo efectos en las burocracias públicas, que también atravesaron un proceso de redefinición interna: por ejemplo, Enrique Rodríguez fue designado ministro de Producción de la Ciudad, y si bien continuó desempeñándose como director de la CBAS, sus nuevas funciones afectaron su cotidianidad en el organismo.

La Coordinadora Multisectorial Sudoeste ${ }^{8}$ continuó ejerciendo una resistencia activa al proyecto. En el año 2006, durante la Copa Davis, desplegaron banderas en las tribunas con la inscripción "Sí a la Copa Davis, sí al hospital, sí al Parque de las Victorias, no al polo farmacéutico". Pocos meses después, realizaron cortes en las avenidas que rodeaban el predio en donde se iba a emplazar el polo, con el fin de manifestar su oposición. Los laboratorios farmacéuticos, por su parte, presionaron al Ejecutivo local para que el proyecto avanzara. También intentaron influir en la opinión pública a partir de la publicación de notas en diarios nacionales que explicaban el proyecto, las inversiones que demandaría y sus supuestas ventajas para el desarrollo de la zona sur.

En julio de 2006 el Gobierno de la Ciudad firmó el boleto de compra-venta del predio con los laboratorios. La escrituración se produjo recién un año después, durante la campaña electoral de Jorge Telerman, que buscaba renovar su cargo como jefe de Gobierno. Ese era el año límite establecido por la ANMAT para la readecuación de los laboratorios a las regulaciones para la fabricación y control de medicamentos. En este sentido, aunque COOPERALA presionó durante todo el período para que el polo se concretara, las resistencias territoriales, los cambios en la coyuntura política y las variaciones producidas en el interior de las burocracias estatales afectaron los tiempos de ejecución del proyecto.

\section{La Coordinadora}

Multisectorial Sudoeste fue una organización surgida a partir de rearticulación de los grupos que rechazaban el emplazamiento del polo farmacéutico. Nucleaba al Movimiento por el Hospital de Lugano, Soldati y villa Riachuelo, vecinos autoconvocados por la urbanización de villa 20 y autoconvocados por la defensa de los espacios públicos y en contra de la construcción del polo farmacéutico. A la resistencia encabezada por la coordinadora, se sumaron nuevos grupos, como el Movimiento de Trabajadores Desocupados de Villa 20 y la Unión de Trabajadores Desocupados. 
9. Esta dependencia fue creada durante esa gestión con el fin de promover la realización de inversiones en la ciudad de Buenos Aires. Muchas de esas inversiones tuvieron anclaje urbano, como por ejemplo el Polo Tecnológico de Parque Patricios, que fue uno de los proyectos más promocionados durante el período de Mauricio Macri como jefe de Gobierno.

10. Cabe señalar que el estudio de impacto ambiental fue realizado por un ingeniero vinculado con la industria farmacéutica y asesor de COOPERALA.

\section{El polo durante la gestión macrista: nuevos cambios en el contexto político y atrasos}

La trayectoria del polo evidencia que estas intervenciones se van conformando en un entramado complejo de intereses, disputas y contextos, que inciden en la forma y condiciones de materialización de los proyectos, así como en su viabilidad. Esto se hizo aún más evidente en los años siguientes, cuando se produjeron nuevos cambios políticos y económicos. Entre la escrituración de los terrenos del polo — producida en el año 2007-y el comienzo de las obras -en el año 2012-, pasaron cinco años en los que los laboratorios debieron atravesar distintos contratiempos. Con la asunción del jefe de Gobierno Mauricio Macri, de orientación neoliberal, en diciembre de 2007, se llevó adelante un profundo proceso de modificación de la estructura burocrática del Estado, que implicó la creación de nuevas dependencias, la reasignación de funciones entre los distintos organismos ejecutivos del Estado y el nombramiento de nuevos funcionarios en las entidades vinculadas con el proyecto.

En este contexto, COOPERALA solicitó formalmente la intervención del Ministerio de Desarrollo Económico de la ciudad como facilitador del emprendimiento y referente por parte del Ejecutivo porteño, que se involucró a través del Centro de Atención al Inversor ${ }^{9}$ (CAI). Esa entidad funcionó de enlace entre COOPERALA y el resto de las dependencias estatales con el propósito de acelerar los tiempos de ejecución del proyecto. Así, durante el año 2009, el CAI realizó gestiones ante la Agencia de Protección Ambiental de la ciudad (AprA), con el fin de que el polo obtuviera un certificado de factibilidad ambiental, que era uno de los requisitos para comenzar las obras.

El estudio de impacto ambiental fue puesto en debate en 2010, en una audiencia pública convocada por la Agencia de Protección Ambiental de la ciudad (GCBA-Subsecretaría de Atención Ciudadana, 2010). En ese ámbito, los representantes de COOPERALA y los laboratorios farmacéuticos defendieron el estudio realizado, destacaron que la farmacéutica era una industria limpia y enfatizaron los beneficios que el emprendimiento iba a producir en la zona. La Multisectorial Sudoeste, por su parte, puso en duda la objetividad del estudio ${ }^{10}$, enfatizó el perfil contaminante de la industria, señaló la emergencia ambiental en el área y criticó los escasos avances en la construcción del hospital y la reurbanización de la villa 20. La Agencia de Protección Ambiental habilitó el inicio de las obras a partir del otorgamiento del certificado de Aptitud Ambiental, pero condicionó la habilitación de los laboratorios a 
un nuevo estudio que evaluara los efectos sinérgicos generados por las distintas plantas. Esto fue recibido con cierta disconformidad por parte de los laboratorios, que visualizaron ese requisito como una nueva traba burocrática.

El Centro de Atención al Inversor realizó reuniones con las empresas proveedoras de servicios, medió para que los laboratorios obtuvieran sus certificados de factibilidad de servicios, realizó el seguimiento de los trámites para obtener los certificados de impacto ambiental y de obra. Con el propósito de apoyar el emprendimiento, el Banco Ciudad creó una línea específica de créditos para financiar obras de infraestructura y con la posibilidad de extenderse a la construcción de las plantas. Se estimaba que los primeros tres laboratorios podrían instalarse en el año 2013 y que en 2014 el Polo estaría funcionando plenamente. Sin embargo, el proyecto atravesó nuevos obstáculos que volvieron a frenar su desarrollo. Una de las principales dificultades fueron las aprobaciones de los servicios de gas, agua y electricidad. Las gestiones con las empresas Metrogas, AySA y Edesur demoraron más de lo esperado, y recién en el año 2012 estuvieron dadas las condiciones para construir las redes de infraestructura. Como se verá en el próximo apartado, a esto se sumaron otro tipo de dificultades, relacionadas con cambios en la coyuntura económica e industrial del sector farmacéutico, así como en la situación y proyección de los laboratorios.

\section{Diferencias entre los laboratorios y un proyecto a la deriva}

Entre las primeras definiciones del proyecto del polo y el comienzo de obras pasaron doce años. A lo largo de ese período, las necesidades, condiciones y recursos de las empresas que habían apoyado la iniciativa fueron cambiando. En el año 2018, solo uno de los once laboratorios involucrados construyó su planta y se encuentra en funcionamiento (ver imagen 2).

Por un lado, se produjeron recambios en el grupo que originalmente estuvo interesado en la propuesta, lo que trajo aparejados intereses y objetivos diversos en torno al proyecto. Esta cuestión se vio reforzada por los avances que realizó cada laboratorio en la construcción o adecuación de su planta a las regulaciones de la $\mathrm{ANMAT}^{11}$, que había sido uno de los principales problemas que se buscaba resolver a través del emprendimiento. De este modo, cuando el polo tuvo la oportunidad de materializarse, las circunstancias particulares de sus promotores se habían modificado. Esto se tradujo en disidencias internas con respecto a las
11. Como se señaló en los apartados anteriores, los laboratorios tenían un plazo definido para readecuar sus plantas a las regulaciones de la ANMAT. Las demoras en el proyecto de polo provocaron que construyeran sus plantas en otras localizaciones o readecuaran las que tenían en funcionamiento. 
Imagen 2. Planta de Química Montepellier, Polo Farmacéutico, Comuna 8. Año 2017. Fuente: María Soledad ARQueros MEJICA Y CARolina GonzÁLEZ REDONDO
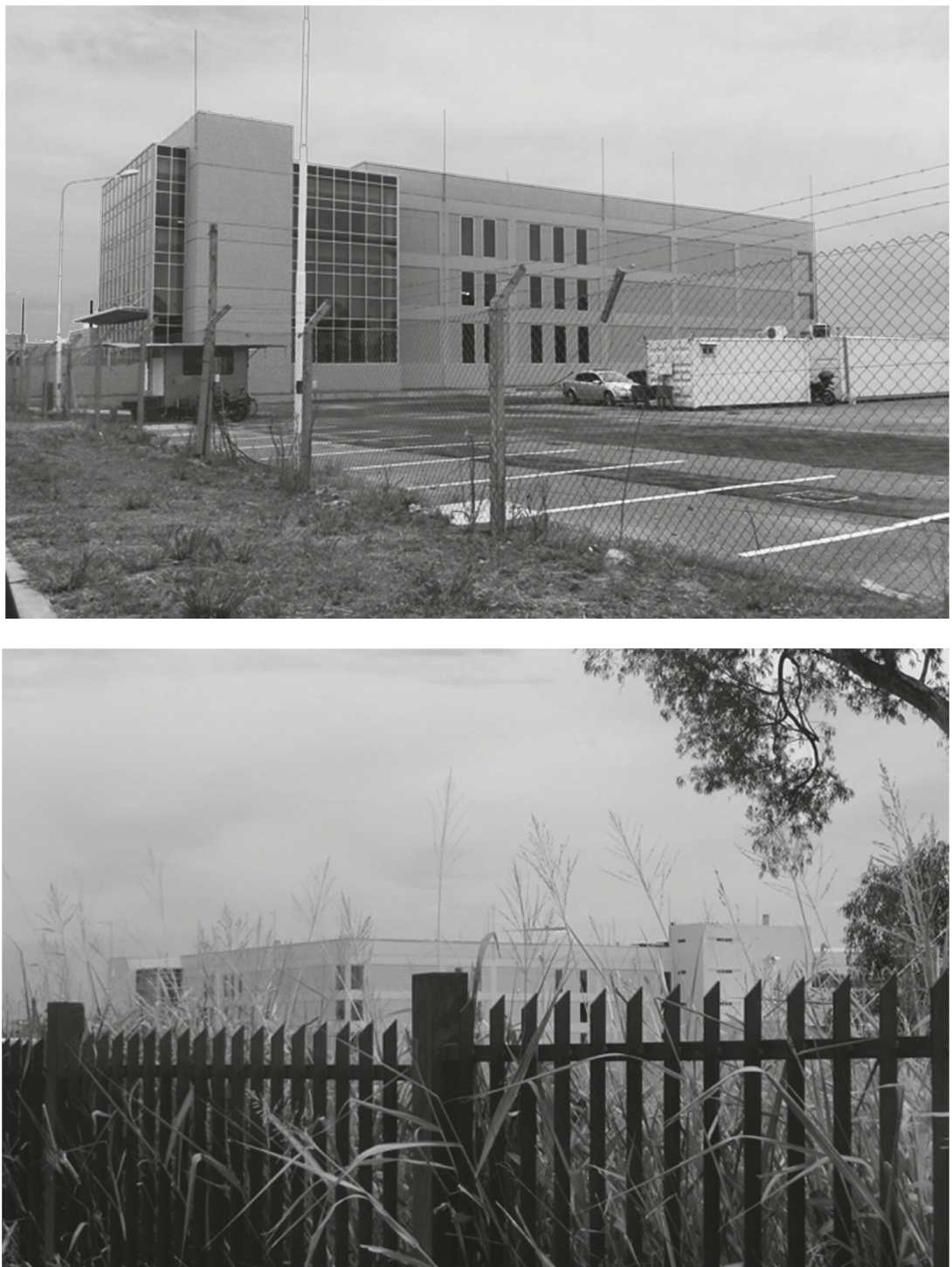
finalidades del proyecto, así como criterios distintos sobre el modo de emprender acciones de producción y de investigación y desarrollo (JuNCAL ET ÁL., 2013).

Por otro lado, en los primeros años de la década de 2010, los laboratorios nacionales mantuvieron una fuerte disputa con el Gobierno Nacional, ya que, desde su punto de vista, este tomó medidas que afectaron la rentabilidad del sector. En este sentido, a partir del año 2003 crecieron la producción, ventas y exportaciones de los laboratorios nacionales, pero - según el sector farmacéutico- las regulaciones de precios produjeron retrasos con respecto a los $\operatorname{costos}^{12}$. Sobre este aspecto, Juan Carlos Bagó — cuya firma estaba involucrada en el proyecto del polo- señalaba que "el 2013 ha sido un año difícil para toda la industria por el retraso de los precios y el crecimiento de los costos, que han limitado el crecimiento y la inversión” (Pharma Baires, 9 de diciembre de 2013).

En este contexto, algunas de las empresas que estaban involucradas en el proyecto quebraron o fueron absorbidas. Otras decidieron dejar en suspenso la construcción de sus plantas argumentando que estaban en una situación financiera desfavorable y que no habían logrado unificar criterios sobre los fines y modos de funcionamiento del polo. Paradójicamente, solo Química Montpellier, del grupo Bagó, construyó su planta. Según los hermanos Bagó, el proyecto los agarró en el baile, le "pusieron el hombro" y hasta se "endeudaron para concretarlo" (Pharma Baires, 30 de noviembre de 2015).

\section{Algunas reflexiones provisorias}

Este artículo focalizó la atención en las formas de coordinación y organización entre organizaciones estatales, empresariales y sociales en torno a un proyecto de intervención urbana. Específicamente, retomó el caso del polo farmacéutico promovido por un grupo de laboratorios y varias agencias del Estado en el marco de la política de desarrollo de la Comuna 8. El proyecto, que fue presentado en 2004, recibió rápidamente el apoyo del Ejecutivo local y de agencias del gobierno nacional, por lo que su materialización parecía inexorable. Sin embargo, en 2018, permanece inconcluso: solo uno de los once laboratorios involucrados logró construir su planta.

El caso analizado permite dialogar así con otros enfoques que tuvieron una incidencia notable en el estudio de los procesos de desarrollo y transformación de las ciudades.
12. La industria farmacéutica local depende de la importación de principios activos, lo que le supone costos altos y un déficit comercial estructural. Asimismo, en este sector se logró que los salarios aumentaran al ritmo de la inflación, pero la imposibilidad de los laboratorios de trasladar esos incrementos a los precios afectó - según las cámaras empresariales-su tasa de ganancia. 
En particular, con algunos elementos de las tesis de las máquinas de crecimiento y los regímenes urbanos, referidos a los vínculos entre actores estatales y no estatales, y al papel que juegan las condiciones de contexto en la orientación y resultados de este tipo de iniciativas. La experiencia del polo evidencia que estos proyectos están sujetos a múltiples mediaciones. Específicamente, pone de relieve que se desarrollan en un complejo entramado de intereses, disputas y contextos, que los condicionan y afectan su viabilidad.

Un elemento central que se deprende del análisis está vinculado con la diversidad de posturas que asumen los diferentes poderes y agencias de un mismo poder del Estado. Esto se reflejó en los cursos de acción desplegados por las distintas burocracias, que tendieron a diferenciarse y, por momentos, a contradecirse unas con otras. Así, por ejemplo, mientras la Corporación Buenos Aires Sur promovía el polo, el Instituto de la Vivienda de la Ciudad realizaba gestiones para afectar esos terrenos a la reurbanización de la villa 20. Esto también se reflejó en el nivel nacional, cuando el Ministerio de Ciencia y Técnica se involucró de manera activa en el proyecto y, al mismo tiempo, el Ministerio de Economía mantuvo una disputa con el sector farmacéutico, que según los laboratorios afectó sus posibilidades de inversión en el polo. Esta diversidad de posturas en el interior del Estado tiende a interrogar el supuesto de que su acción está siempre subordinada a los intereses de los sectores empresariales, aun cuando se asume — como lo hacemos aquí-que su función principal es garantizar la reproducción de la relación social capitalista.

Esa diversidad de posturas e intereses se observa también en el territorio, que constituye otro ámbito de la disputa política. La incidencia de ese entramado complejo de actores en la orientación de las intervenciones y la materialización del proyecto pone de relieve el papel que juegan estos grupos, cuya acción y capacidad de veto tienden a ser desestimadas en los enfoques analíticos reseñados. En este sentido, el proceso analizado da cuenta de que las acciones desplegadas por aquellos sectores (que en términos de la máquina de crecimiento podrían denominarse contracoaliciones) tuvieron como correlato una actualización del marco jurídico-político, a partir de la sanción de las leyes de reurbanización de villa 20 y de construcción del hospital de villa Lugano. A su vez, muestra que las resistencias organizadas en el territorio fueron demorando instancias claves para la ejecución del proyecto, 
como la sanción de la ley para su emplazamiento, aspecto que afectó sus oportunidades de materialización en el corto plazo.

Esto fue reforzado por las variaciones en el contexto político de la ciudad y en la coyuntura económica de los laboratorios. Con cada cambio de gestión de gobierno se modificaron las prioridades de política y las orientaciones de organismos clave. En particular, el cambio de autoridades de la Corporación Buenos Aires Sur implicó que esa entidad, que había jugado un papel clave en el impulso del polo, quedara prácticamente desvinculada de este. Ese lugar fue ocupado por otras organizaciones estatales, que si bien apoyaron el proyecto, no necesariamente lo tuvieron entre sus prioridades. Cuando se cumplieron todos los pasos necesarios para habilitar la construcción de las plantas, la coyuntura económica y las necesidades de los laboratorios se habían modificado, lo que alteró los consensos originales en torno a los objetivos y criterios del polo y puso en jaque la totalidad del proyecto. Esto evidencia, en diálogo con otros enfoques analíticos, que el contexto en el que se desarrollan estas iniciativas forma parte integrante de ellas y ejerce un rol clave en las formas que asumen, así como en sus posibilidades de concreción.

Lo hasta aquí señalado habilita una reflexión sobre las diferentes temporalidades de los actores y sus efectos en estos procesos de intervención urbana. En este sentido, el proyecto del polo farmacéutico dependía para materializarse de la coordinación de un espectro relativamente amplio de dependencias y organizaciones, estatales y no estatales, que tienen sus propias lógicas y ritmos de producción. Para aprobar la ley que habilitaba su construcción, era necesario pasar por el mecanismo de doble lectura y realizar las audiencias públicas correspondientes. Una vez aprobada esa ley, los laboratorios debían obtener permisos y habilitaciones emitidas por organismos públicos (como la Agencia de Protección Ambiental) y privados (como algunas empresas prestadoras de servicios), que demoraron más tiempo del previsto. Cuando estuvo todo listo para comenzar a construir las plantas, las circunstancias particulares de los laboratorios habían cambiado, por lo que varios de ellos habían desistido de continuar con el proyecto. De esta manera, las diversas organizaciones estatales y no estatales tuvieron temporalidades propias, que tendieron a desfasarse entre sí y afectaron la viabilidad del proyecto. 


\section{Bibliografía}

ABRUTZKY, R., BRAMUGLIA, C. y GODIO, C. (2015). "El perfil de la industria farmacéutica de la Argentina. Interrogantes a mediano plazo”. Ciencia, Docencia y Tecnología, 51 (noviembre): $102-30$

ACUÑA, C. (1994). "El análisis de la burguesía como actor político”. Ponencia presentada en el IV Seminario Internacional del Grupo de Trabajo 'Empresarios y Estado', CLACSO, México, 28-30 de septiembre.

ARQUEROS MEJICA, M. S. (2018) La política de desarrollo de la Comuna 8 de la ciudad de Buenos Aires (1996-2015). Tesis de doctorado inédita en Ciencias Sociales, Facultad de Ciencias Sociales, Universidad de Buenos Aires.

ARQUEROS MEJICA, M. S. (2014) Procesos de producción social del hábitat y políticas públicas en las villas de la ciudad de Buenos Aires. El caso de Barrio INTA. Tesis inédita para acceder al grado de Magíster en Economía Urbana, Universidad Torcuato Di Tella.

AZPIAZU, D. (1999). "La industria farmacéutica. Las estructuras oligopólicas frente a la desregulación y la apertura de la economía”. En La desregulación de los mercados. Paradigmas e inequidades de las políticas del neoliberalismo. Daniel Azpiazu, Graciela Gutman y Adolfo Vispo (compiladores), 164-269. Buenos Aires: Grupo Editorial Norma/FLACSO.

BELTRÁN, G. (2011). "Las paradojas de la acción empresaria. Las asociaciones del empresariado argentino y la persistencia de las reformas estructurales”. En Los años de Menem: la construcción del orden neoliberal Alfredo Pucciarelli (coordinador), 221-262. Buenos Aires: Siglo XXI editores.

BROWN, M. y ERIE, S. (1984). "Poder y administración: paradigmas alternativos para el análisis de la autonomía burocrática”. En Teoría de la burocracia estatal Oscar Ozslak (compilador). Buenos Aires: Paidós.

CLICHEVSKY, N. (1996) Política social urbana. Normativa y configuración de la ciudad. Buenos Aires: Espacio Editores.

COCHRANE, A. (1999). "Redefining urban politics for the thwenty-first century". En The urban growth machine. Critical perspectives two decades later. Compilado por Andrew E. G. Jonas \& David Wilson, 109-124. New York: State University of New York Press.

CUENYA, B. (2006) Large urban projects and social actors. Forces supporting and oppsing the production process of the Retiro Project, Buenos Aires 1991-2001. Tesis doctoral, TU Deft, Faculty of Architecture, Chairof Spatial Planning. 
CUENYA, B. (2005). "Grandes proyectos y teorías sobre la nueva política urbana en la era de la globalización. Reflexiones a partir de la experiencia de la ciudad de Buenos Aires”. En Fragmentos sociales. Problemas urbanos de la Argentina. Beatriz Cuenya, Hilda Herzer y Carlos Fidel (compiladores), 89-110. Buenos Aires: Siglo XXI.

CUENYA, B. y CORRAL, M. (2011). "Empresarialismo, economía del suelo y grandes proyectos urbanos: el modelo de Puerto Madero”. Eure, 111 (mayo): 25-45.

DOSSI, M. y LISSIN, L. (2011). "La acción empresarial organizada: propuesta de abordaje para el estudio del empresariado”. Revista Mexicana de Sociología, 73 (julio-septiembre): 415-443.

GOICOCHEA, M. E. (2014). "La ciudad de Buenos Aires como ámbito y objeto de negocios. Reflexiones en torno a la gestión urbana del Distrito Tecnológico de Parque Patricios”. Quid 16, 4 (noviembre 2014-octubre 2015): 161-85.

GUEVARA, T. (2013). "La renovación como estrategia de desarrollo urbano en Buenos Aires (1996-2011)”. Apuntes, 2 (julio-diciembre): 68-79.

HARVEY, D. (2007) Espacios del capital. Hacia una geografía crítica. México: Akal.

LASCOUMES, P. y LE GALÉS, P. (2014) Sociología de la acción pública. México: CEDUA-COLMEX.

LOGAN, J. R. y MOLOTCH, H. (2015 [1984]). “La ciudad como máquina de crecimiento”. En El mercado contra la ciudad. Globalización, gentrificación y políticas urbanas, editado por el Observatorio Metropolitano de Madrid, 157-210. Madrid: Traficantes de sueños.

MENAZZI, L. (2015). "Entramado público-privado en la construcción de ciudad. Obra pública y regulaciones en la ciudad de Buenos Aires”. Revista Ciudades [106], pp. 45-52.

MOLOTCH, H. (1999). "Growth machine links: un, down, and across". En The urban growth machine. Critical perspectives two decades later. Compilado por Andrew E. G. Jonas \& David Wilson, 247-266. New York: State University of New York Press.

OSZLAK, O. (2006). "Burocracia estatal: política y políticas públicas". Revista POSTdata [11], pp. 11-56.

OSZLAK, O. y O’DONNELL, G. (2008 [1976]). "Estado y políticas estatales en América Latina: hacia una estrategia de investigación”. En Lecturas sobre el Estado y las políticas públicas: retomando el debate de ayer para fortalecer el actual. Carlos Acuña (compilador), 203-238. Buenos Aires: Jefatura de Gabinete de Ministros.

RODRÍGUEZ, M. C. y DI VIRGILIO, M. (2011). “Coordenadas para el análisis de las políticas urbanas: un enfoque territorial”. En Caleidoscopio de las políticas territoriales. Un rompecabezas para armar. M. Carla Rodríguez y Mercedes Di Virgilio (compiladoras), 17-46. Buenos Aires: Prometeo libros. 
PANAIA, M. (2004) El sector de la construcción: un proceso de industrialización inconcluso. Buenos Aires: Nobuko.

PORTES, A. (1999) La economía informal y sus paradojas. En Informalidad y exclusión social. Carpio J., Klein E. e Novacovsky I. (editores). Buenos Aires: SIEMPRO/OIT/Fondo de Cultura Económica de Argentina.

RASPALL, T. (2014) El rol de los actores en la configuración de la oferta de vivienda nueva en la ciudad de Buenos Aires durante los primeros años de la posconvertibilidad. El caso del barrio de Caballito, 2003-2008. Tesis de maestría inédita. Instituto de Altos Estudios Sociales, Universidad Nacional de San Martín, Buenos Aires.

SCHTEINGART, M. (1979). "Sector inmobiliario capitalista y formas de apropiación del suelo urbano: el caso de México”. Demografía y Sociedad, 13 [4].

SOCOLOFF, I. (2015). "Financiamiento global y centros comerciales en Buenos Aires: un estudio del caso IRSA”. Revista INVI, 30 [84], pp. 151-177.

STONE, C. (1993). "Urban Regimes and the Capacity to Govern: An Political Economy Approach. Journal of Urban Affairs, 5 [1], pp. 1-28.

STONE, C. (1980). "Systemic power and community decision making: a restatement of stratification theory”. The American Political Science Review, 74 (diciembre): 978-990.

SVAMPA, M. y VIALE, E. (2014) Maldesarrollo. La Argentina del extractivismo y el despojo. Buenos Aires: Katz.

THWAITES REY, M. (2005). “Estado: ¿qué Estado?”. En Entre tecnócratas globalizados y políticos clientelistas. Derrotero del ajuste neoliberal en el Estado argentino. Mabel Thwaites Rey y Andrea López (compiladoras), 19-40. Buenos Aires: Prometeo libros.

YUJNOVSKY, O. (1984) Claves políticas del problema habitacional argentino 1955-1981. Buenos Aires: Grupo Editor Latinoamericano.

YUJNOVSKY, O. y CLICHEVSKY, N. (1978). "El sector concentrado de la construcción en la Argentina”. Revista Latinoamericana de Planificación [47-48]. 


\section{Fuentes}

Equipo editorial (2013). "Un año difícil para la industria farmacéutica”. Pharma Baires. 9 de diciembre.

Equipo editorial (2015). "Otro año difícil pero con optimismo". Pharma Baires. 30 de noviembre.

GCBA (2004). "Programa General de Acción de Gobierno 2005-2007”. Buenos Aires: Gobierno de la Ciudad de Buenos Aires.

GCBA-Subsecretaría de Atención Ciudadana (2010). "Audiencia Pública: Análisis del proyecto de Polo Farmacéutico, sito en las avenidas Escala y Fernández de la Cruz". Versión Taquigráfica. Buenos Aires: Jefatura de Gabinete del Gobierno de la Ciudad Autónoma de Buenos Aires.

Juncal, S.; Kohon, F.; Mochi, S. y Rajzman, N. (2013). Análisis de diagnóstico tecnológico sectorial. Complejo productivo farmacéutico. Buenos Aires: Ministerio de Ciencia, Tecnología e Innovación Productiva.

Legislatura Porteña (2005). "Ley 1770: Afecta polígonos a la urbanización de Villa 20. Modifica el Código de Planeamiento. Terrenos afectados a la construcción de vivienda". 11/08/2005. Publicada en el Boletín Oficial Nro. 2281 del 15/09/ 2005.

Legislatura Porteña (2005). "Ley 1769: Dispone la construcción de un Hospital en el barrio de Villa Lugano. Inicio de obras: 12 meses desde la promulgación”. 11/08/2005. Publicada en el Boletín Oficial Nro. 2283 del 16/09/ 2005.

Legislatura Porteña (2005). "Ley 1768: Modificación del Código de Planeamiento Urbano. Incorpora un Polo Farmacéutico”. 11/08/2005. Publicada en el Boletín Oficial Nro. 2.288 del 20/09/2005.

Legislatura Porteña (2000). "Ley 470: Creación de la Corporación Buenos Aires Sur S. E”. 05/08/2000. Publicada en el Boletín Oficial Nro. 1025 del 12/09/2000.

Poder Judicial de la Ciudad Autónoma de Buenos Aires (2005). Expediente judicial 16693/0, caratulado "JUÁREZ SARA ESTEL CONTRA GCBA SOBRE AMPARO (ART. 14 CABA)". Buenos Aires.

Redacción La Nación (2004). "Piden rapidez para el Polo Farmacéutico". En La Nación 11/10/2004. Buenos Aires.

Rodríguez, E. (2005). “Para desarrollar el sur porteño”. En La Nación 12/05/2005. Buenos Aires. 